\title{
Planktic foraminiferal biostratigraphy, paleoecology and chronostratigraphy across the Eocene/Oligocene boundary in northern Tunisia
}

\section{Narjess Karoui-Yaakoub ${ }^{\mathrm{a}, \mathrm{b}}$}

Chaima Grira ${ }^{\mathrm{a}, \mathrm{b}}$

Moncef Saïd Mtimet $^{a}$

Mohamed Hédi Negra

Eustoquio

emolina@unizar.es

aDépartement des Sciences de la Terre, Faculté des Sciences de Bizerte, Université de Carthage, Jarzouna, Bizerte, 7021, Tunisia

bunité de Recherche: Pétrologie Sédimentaire et Cristalline, Université de Tunis El Manar, Tunisia

'Departamento de Ciencias de la Tierra \& IUCA, Universidad de Zaragoza, E-50009, Zaragoza, Spain

${ }^{*}$ Corresponding author

\section{Abstract}

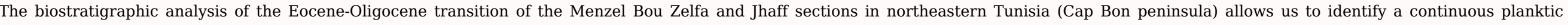

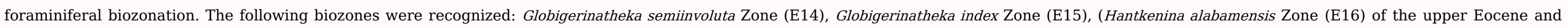

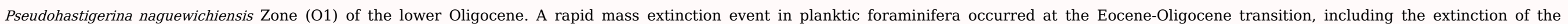

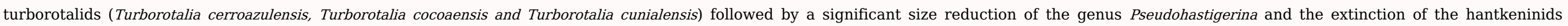

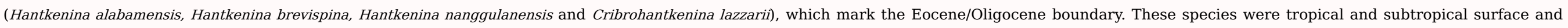

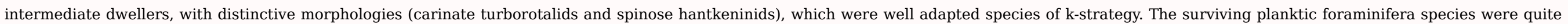

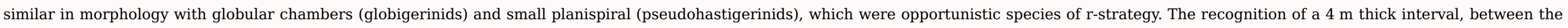

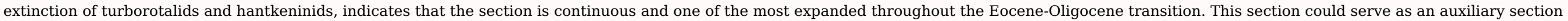
(hypostratotype) for the complete definition of the Global Stratotype Section and Point for the Eocene/Oligocene boundary, which mark the base of the Rupelian Stage.

Keywords: Planktic foraminifera; Eocene/Oligocene; Biostratigraphy; Paleoecology; Chronostratigraphy; Tunisia

\section{Introduction}

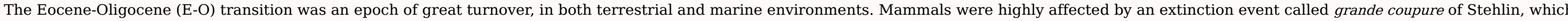

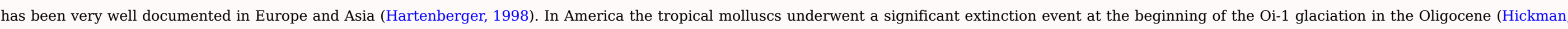

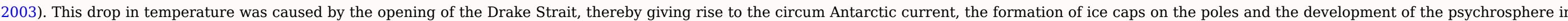

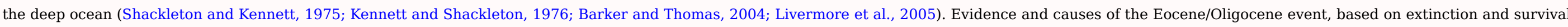
patterns of foraminifera, were reviewed by Molina (2015, see other references herein).

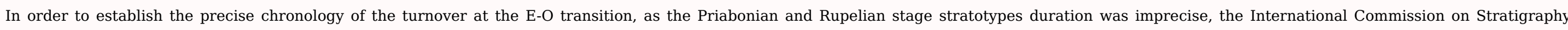

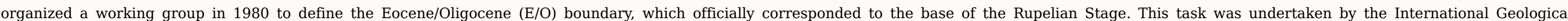




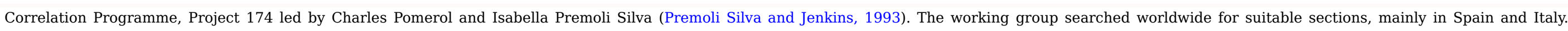

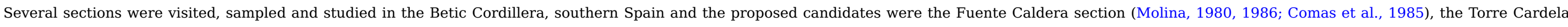

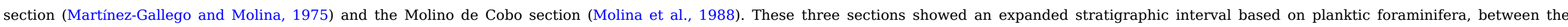

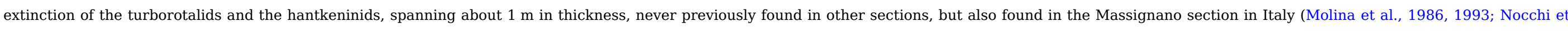

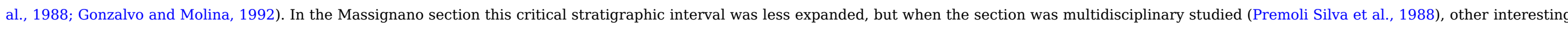

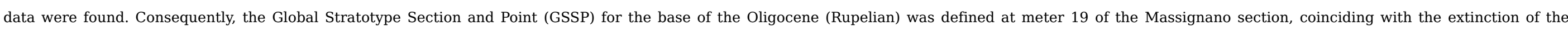
hantkeninids (Premoli Silva and Jenkins, 1993).

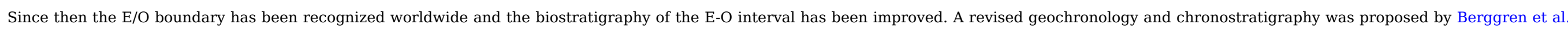

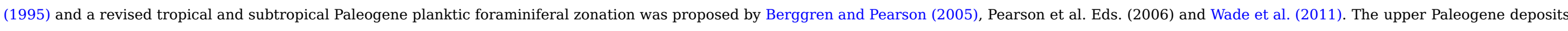

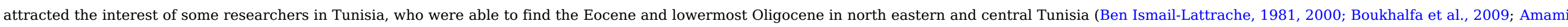

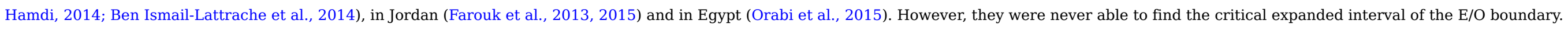

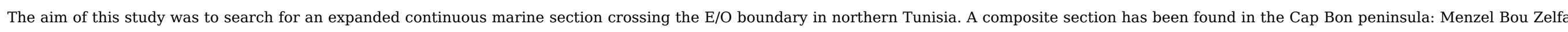

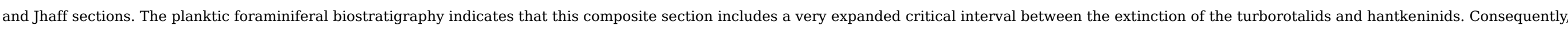
this section is suitable and could be a potential auxiliary section (hypostratotype) to complement the definition of the E/O boundary that was defined in the Massignano section, Italy.

\section{Material and methods}

\subsection{Geological and geographical location}

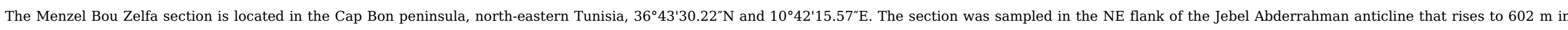

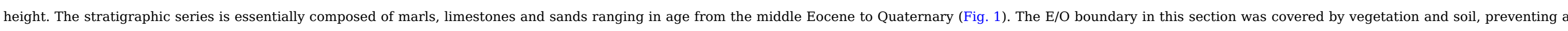

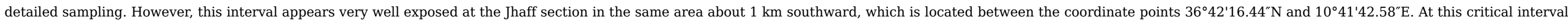
the samples, which are named J 6 to J 13, are located by correlation between samples MBZ 26 and MBZ 25. 


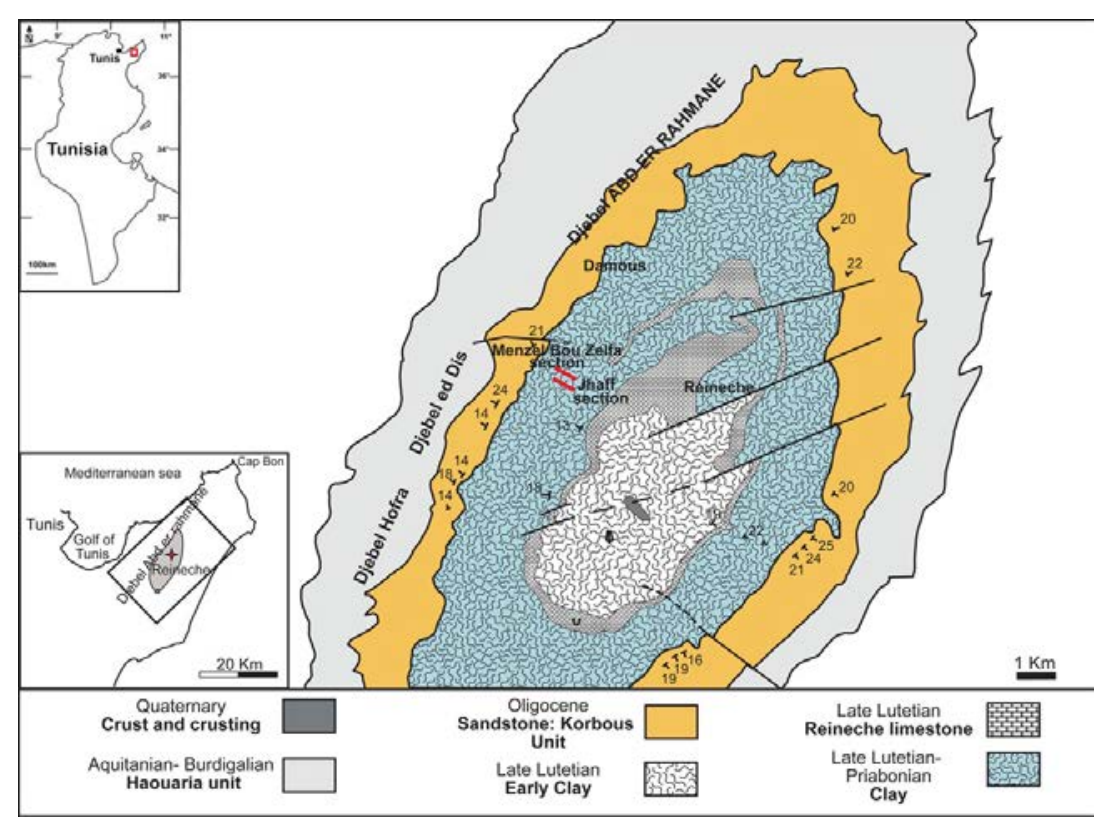

Fig. 1 Geographical and geological location of the Menzel Boy Zelfa and Jhaff sections.

alt-text: Fig. 1

\subsection{Methods}

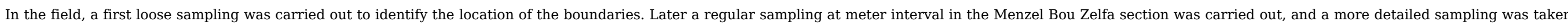

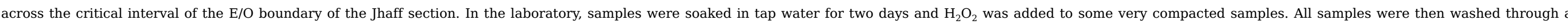

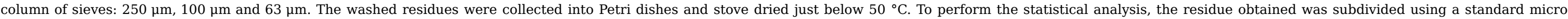

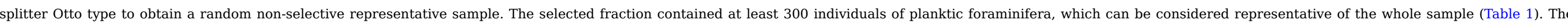

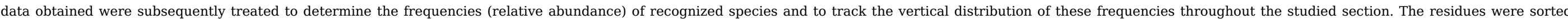
under a binocular microscope to identify the planktic foraminifera species and the rest of the sample was scanned to look for rare species.

Table 1 Table of the quantitative analysis of planktic foraminifera species.

\section{alt-text: Table 1}

Samples

Catapsydrax Catapsydrax Catapsydrax Catapsydrax Catapsydrax Cribrohantkenina Cribrohantkenina Dentoglobigerina Dentoglobigerina dissimilis globiformis howei africanus $\begin{array}{cccc}\text { atapsydrax } & \text { Cribrohantkenina } & \text { Cribrohantkenina } \\ \text { inflata } & \begin{array}{c}\text { Dentoglobige } \\ \text { tripartita }\end{array}\end{array}$

Species

\begin{tabular}{|c|c|c|c|c|c|c|c|c|c|c|c|c|}
\hline & $\begin{array}{l}\text { Catapsydrax } \\
\text { dissimilis }\end{array}$ & $\begin{array}{l}\text { Catapsydrax } \\
\text { globiformis }\end{array}$ & $\begin{array}{l}\text { Catapsydrax } \\
\text { howei }\end{array}$ & $\begin{array}{l}\text { Catapsydrax } \\
\text { africanus }\end{array}$ & $\begin{array}{l}\text { Catapsydrax } \\
\text { unicavus }\end{array}$ & $\begin{array}{c}\text { Cribrohantkenina } \\
\text { lazzari }\end{array}$ & $\begin{array}{c}\text { Cribrohantkenina } \\
\text { inflata }\end{array}$ & $\begin{array}{c}\text { Dentoglobigerina } \\
\text { tripartita }\end{array}$ & $\begin{array}{c}\text { Dentoglobigerina } \\
\text { galavisi }\end{array}$ & $\begin{array}{l}\text { D. pseudo- } \\
\text { venezuelana }\end{array}$ & $\begin{array}{l}\text { Globigerinatheka } \\
\text { semiinvoluta }\end{array}$ & $\begin{array}{l}\text { Globigerinatheka } \\
\text { index }\end{array}$ \\
\hline MBZ 1 & 0 & 0 & 0 & 0 & 0 & 0 & 0 & 0 & 0 & 0 & 0 & 0 \\
\hline MBZ 2 & 0 & 0 & 0 & 0 & 0 & 0 & 0 & 0 & 0 & 0 & 0 & 0 \\
\hline MBZ 3 & 0 & 0 & 0 & 0 & 0 & 0 & 0 & 0 & 0 & 0 & 0 & 0 \\
\hline MBZ 4 & 0 & 0 & 0 & 0 & 0 & 0 & 0 & 0 & 0 & 0 & 0 & 0 \\
\hline MBZ 5 & 0 & 0 & 0 & 0 & 0 & 0 & 0 & 0 & 0 & 0 & 0 & 0 \\
\hline
\end{tabular}




\begin{tabular}{|c|c|c|c|c|c|c|c|c|c|c|c|c|}
\hline MBZ 6 & 0 & 0 & 0 & 0 & 0 & 0 & 0 & 0 & 0 & 0 & 0 & 0 \\
\hline MBZ 7 & 0 & 0 & 0 & 0 & 0 & 0 & 0 & 0 & 0 & 0 & 0 & 0 \\
\hline MBZ 8 & 0 & 0 & 0 & 0 & 0 & 0 & 0 & 0 & 0 & 0 & 0 & 0 \\
\hline MBZ 9 & 0 & 0 & 0 & 0 & 0 & 0 & 0 & 0 & 0 & 0 & 0 & 0 \\
\hline MBZ 10 & 0 & 0 & 0 & 0 & 0 & 0 & 0 & 0 & 0 & 0 & 0 & 0 \\
\hline MBZ 11 & $\mathrm{X}$ & 0 & 0 & 0 & 0,19 & 0 & 0 & 0,38 & 2,2 & 1,7 & 0 & 0 \\
\hline MBZ 12 & $\mathrm{X}$ & 0 & 0 & 0 & 0,2 & 0 & 0 & 0,4 & 0 & 3,5 & 0 & 0 \\
\hline MBZ 13 & 0 & 0 & 0 & 0 & $\mathrm{x}$ & 0 & 0 & 0,3 & 0 & 2,6 & 0 & 0 \\
\hline MBZ 14 & $\mathrm{X}$ & 0 & 0 & 0 & 0,3 & 0 & 0 & 0 & 5,5 & 7,9 & 0 & 0 \\
\hline MBZ 15 & $\mathrm{x}$ & 0 & 0 & 0 & $\mathrm{x}$ & 0 & 0 & 0,6 & 0 & 1,5 & 0 & 0 \\
\hline MBZ 16 & 0 & 0 & 0 & 0 & $\mathrm{x}$ & 0 & 0 & 7,2 & 2,4 & 1,9 & 0 & 0 \\
\hline MBZ 17 & $\mathrm{x}$ & 0 & 0 & 0 & $\mathrm{x}$ & 0 & 0 & 0 & 1,1 & 2,5 & 0 & 0 \\
\hline MBZ 18 & $\mathrm{X}$ & 0 & 0 & 0 & 0 & 0 & 0 & 1,3 & 0 & 7,4 & 0 & 0 \\
\hline MBZ 19 & $\mathrm{X}$ & 0 & 0 & 0 & 0 & 0 & 0 & 0,7 & 0,5 & 1,1 & 0 & 0 \\
\hline MBZ 21 & $\mathrm{x}$ & 0 & 0 & 0 & 0 & 0 & 0 & 0 & 0 & 0 & 0 & 0 \\
\hline MBZ 22 & 0 & 0 & 0 & 0 & 0 & 0 & 0 & 0 & 0,3 & 0 & 0 & 0 \\
\hline MBZ 23 & 0.31 & 0 & 0 & 0 & 0 & 0 & 0 & 0 & 0 & 0 & 0 & 0 \\
\hline MBZ 24 & 0 & 0 & 0 & 0 & 0 & 0 & 0 & 0 & 0 & 0 & 0 & 0 \\
\hline MBZ 25 & $\mathrm{x}$ & 0 & 0 & 0 & 0 & 0 & 0 & 0,3 & 0 & 0 & 0 & 0 \\
\hline Jhaff 13 & 0 & 0 & 0 & 0 & 0,1 & 0 & 0 & 0,18 & 0,5 & 0 & 0 & 0 \\
\hline Jhaff 12 & 0.19 & 0 & 0 & 0 & 0,06 & 0 & 0 & 0,19 & 1,2 & 0,6 & 0 & 0 \\
\hline Jhaff 10 & 0 & 0 & 0 & 0 & 0 & 0 & 0 & 0 & 0,5 & 0 & 0 & 0 \\
\hline Jhaff 9 & 0 & 0 & 0 & 0 & 0 & 0 & 0 & 0 & 0,6 & 0 & 0 & 0 \\
\hline Jhaff 8 & 0 & 0 & 0,05 & 0 & 0,05 & 0,05 & 0 & 0,21 & 0,5 & 0 & 0 & 0 \\
\hline Jhaff 7 & 0 & 0 & 0,1 & 0 & 0 & 0,05 & 0 & 0,26 & 1,4 & 0,9 & 0 & 0 \\
\hline Jhaff 6 & 0 & 0 & 0 & 0 & 0,36 & $\mathrm{X}$ & 0 & 0 & 0,5 & 0,3 & 0 & 0 \\
\hline MBZ 26 & 0 & 0 & 0 & 0 & $\mathrm{X}$ & 0 & 0,27 & 0,27 & 0 & 2,6 & 0 & 0 \\
\hline MBZ 27 & 0 & 0 & 0,8 & 0 & 0 & $\mathrm{X}$ & $\mathrm{X}$ & 0 & 0 & 2,5 & 0 & 0 \\
\hline MBZ 28 & 0 & 0 & 0,3 & 0 & $\mathrm{X}$ & 0 & 0 & 0 & 0 & 0 & 0 & 0,8 \\
\hline MBZ 29 & 0 & 0.15 & 0,3 & 0 & 0 & 0 & 0 & 0 & 0 & 0,9 & 0 & 0,1 \\
\hline MBZ 30 & 0 & 0.32 & 0,32 & 0,3 & 0 & 0 & $\mathrm{X}$ & 2,9 & 4,5 & 5,8 & 1,6 & 1,9 \\
\hline
\end{tabular}




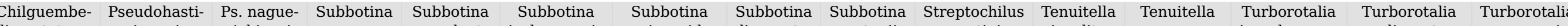

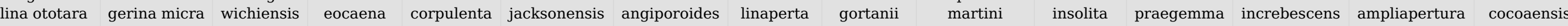

\begin{tabular}{|c|c|c|c|c|c|c|c|c|c|c|c|c|c|c|c|}
\hline $\begin{array}{l}\text { MBZ } \\
1\end{array}$ & 0 & 0 & 0 & 0 & 0 & 0 & 0 & 0 & 0 & 0 & 0 & 0 & 0 & 0 & 0 \\
\hline $\begin{array}{l}\text { MBZ } \\
2\end{array}$ & 0 & 0 & 0 & 0 & 0 & 0 & 0 & 0 & 0 & 0 & 0 & 0 & 0 & 0 & 0 \\
\hline $\begin{array}{l}\text { MBZ } \\
3\end{array}$ & 0 & 0 & 0 & 0 & 0 & 0 & 0 & 0 & 0 & 0 & 0 & 0 & 0 & 0 & 0 \\
\hline $\begin{array}{l}\text { MBZ } \\
4\end{array}$ & 0 & 0 & 0 & 0 & 0 & 0 & 0 & 0 & 0 & 0 & 0 & 0 & 0 & 0 & 0 \\
\hline $\begin{array}{l}\text { MBZ } \\
5\end{array}$ & 0 & 0 & 0 & 0 & 0 & 0 & 0 & 0 & 0 & 0 & 0 & 0 & 0 & 0 & 0 \\
\hline $\begin{array}{l}\text { MBZ } \\
6\end{array}$ & 0 & 0 & 0 & 0 & 0 & 0 & 0 & 0 & 0 & 0 & 0 & 0 & 0 & 0 & 0 \\
\hline $\begin{array}{l}\text { MBZ } \\
7\end{array}$ & 0 & 0 & 0 & 0 & 0 & 0 & 0 & 0 & 0 & 0 & 0 & 0 & 0 & 0 & 0 \\
\hline $\begin{array}{l}\text { MBZ } \\
8\end{array}$ & 0 & 0 & 0 & 0 & 0 & 0 & 0 & 0 & 0 & 0 & 0 & 0 & 0 & 0 & 0 \\
\hline $\begin{array}{l}\text { MBZ } \\
9\end{array}$ & 0 & 0 & 0 & 0 & 0 & 0 & 0 & 0 & 0 & 0 & 0 & 0 & 0 & 0 & 0 \\
\hline $\begin{array}{l}\text { MBZ } \\
10\end{array}$ & 0 & 0 & 0 & 0 & 0 & 0 & 0 & 0 & 0 & 0 & 0 & 0 & 0 & 0 & 0 \\
\hline $\begin{array}{l}\text { MBZ } \\
11\end{array}$ & 2,4 & 9,5 & 2,4 & 4,94 & 6,65 & $\mathrm{X}$ & 0,76 & 0 & 0 & 4,94 & 0 & 0,57 & 0,95 & $\mathrm{X}$ & 0 \\
\hline $\begin{array}{l}\text { MBZ } \\
12\end{array}$ & 6,9 & 14,6 & 8,003 & 4,60 & 3,47 & $\mathrm{X}$ & 1,77 & 0 & 0 & 8,4 & 0 & 5,09 & 0,24 & 0,4 & 0 \\
\hline $\begin{array}{l}\text { MBZ } \\
13\end{array}$ & 14,5 & 10,7 & 2,6 & 5,37 & 3,22 & $\mathrm{X}$ & 0 & 0 & $\mathrm{X}$ & 11,11 & 0 & 0,89 & 0,17 & 0,71 & 0 \\
\hline $\begin{array}{l}\text { MBZ } \\
14\end{array}$ & 3,9 & 0,7 & 1,5 & 3,9 & 5,53 & $\mathrm{X}$ & 0,39 & 0 & 0 & 2,76 & 0 & 0 & 0 & 1,58 & 0 \\
\hline $\begin{array}{l}\text { MBZ } \\
15\end{array}$ & 10,8 & 10,4 & 12,6 & 5,68 & 3,18 & $\mathrm{X}$ & 4,2 & 0 & 0 & 9,32 & 0 & 0,79 & 0,45 & $\mathrm{X}$ & 0 \\
\hline $\begin{array}{l}\text { MBZ } \\
16\end{array}$ & 2,4 & 6,3 & 7,7 & 5,82 & 15,53 & $\mathrm{X}$ & $\mathrm{X}$ & 0 & $\mathrm{X}$ & 1,94 & 0 & 1,94 & 4,36 & 4,36 & 0 \\
\hline $\begin{array}{l}\text { MBZ } \\
17\end{array}$ & 3,3 & 5,3 & 8,3 & 8,65 & 7,26 & $\mathrm{X}$ & $\mathrm{X}$ & $\mathrm{X}$ & 0 & 2,51 & 0 & 3,91 & 1,95 & 1,11 & 0 \\
\hline $\begin{array}{l}\text { MBZ } \\
18\end{array}$ & 10,2 & 6,4 & 7,8 & 1,36 & 3,4 & $\mathrm{X}$ & 0 & $\mathrm{X}$ & $\mathrm{X}$ & 3,74 & 0 & 1,02 & 2,38 & 1,7 & 0 \\
\hline $\begin{array}{l}\text { MBZ } \\
19\end{array}$ & 11,9 & 9,04 & 7,5 & 2,95 & 8,11 & $\mathrm{X}$ & $\mathrm{X}$ & 0 & 0 & 9,04 & 0 & 0 & 1,6 & 3,5 & 0 \\
\hline
\end{tabular}




\begin{tabular}{|c|c|c|c|c|c|c|c|c|c|c|c|c|c|c|c|}
\hline $\begin{array}{l}\text { MBZ } \\
21\end{array}$ & 18,4 & 9,7 & 7,1 & 5,88 & 5,62 & 0 & 0 & $\mathrm{X}$ & $\mathrm{x}$ & 8,95 & 0 & 0 & 1,27 & $\mathrm{X}$ & 0 \\
\hline $\begin{array}{l}\text { MBZ } \\
22\end{array}$ & 5,1 & 10,2 & 10,6 & 3,85 & 5,78 & 0 & 0 & 0 & 0 & 9,64 & 0 & 0 & 1,92 & 0 & 0 \\
\hline $\begin{array}{l}\text { MBZ } \\
23\end{array}$ & 10,1 & 10,1 & 12,6 & 1,89 & 2,84 & $\mathrm{X}$ & $\mathrm{X}$ & 0 & 0 & 6,01 & 0 & 1,58 & 0,94 & 0,63 & 0 \\
\hline $\begin{array}{l}\text { MBZ } \\
24\end{array}$ & 21,7 & 8,1 & 15,9 & 1,55 & 1,94 & $\mathrm{X}$ & $\mathrm{X}$ & $\mathrm{X}$ & $\mathrm{X}$ & 12,06 & 0 & 0 & 0 & $\mathrm{X}$ & 0 \\
\hline $\begin{array}{l}\text { MBZ } \\
25\end{array}$ & 26,4 & 4 & 12 & 1,84 & 4,3 & $\mathrm{X}$ & $\mathrm{X}$ & 0 & 0,61 & 15,08 & 0 & 0 & 1,23 & $\mathrm{X}$ & 0 \\
\hline $\begin{array}{l}\text { Jhaff } \\
13\end{array}$ & 5,4 & 12,1 & 12,1 & 2 & 2,5 & 0 & 11,29 & 0 & 0,25 & 4,13 & 0 & 0 & 0,12 & 0,5 & 0 \\
\hline $\begin{array}{l}\text { Jhaff } \\
12\end{array}$ & 5,3 & 9,08 & 9,6 & 4,54 & 4,28 & 0 & 7,39 & 0 & 0,38 & 4,41 & 0 & 0 & 0 & 1,94 & 0 \\
\hline $\begin{array}{l}\text { Jhaff } \\
10\end{array}$ & 1,5 & 10,3 & 9,8 & 1,55 & 2,78 & 0 & 5,79 & 0 & 0,33 & 1,33 & 0 & 0 & 0,1 & 0,5 & 0 \\
\hline $\begin{array}{l}\text { Jhaff } \\
9\end{array}$ & 5,1 & 9,6 & 12,5 & 3,59 & 4,35 & 0 & 5,38 & 0 & 0,26 & 2,55 & 0 & 0 & 0,2 & 0,53 & 0 \\
\hline $\begin{array}{l}\text { Jhaff } \\
8\end{array}$ & 7,4 & 15,1 & 10,9 & 4,11 & 4,16 & 0 & 0,16 & 0 & 0,1 & 1,44 & 0 & 2,51 & 0,1 & 0,32 & 0 \\
\hline $\begin{array}{l}\text { Jhaff } \\
7\end{array}$ & 4,4 & 7,07 & 7,02 & 4,2 & 6,92 & 0,62 & 0,57 & 0,2 & 0,15 & 1,35 & 0 & 0,52 & 0,5 & 0,57 & 0 \\
\hline $\begin{array}{l}\text { Jhaff } \\
6\end{array}$ & 9,6 & 20,3 & 13,89 & 3,52 & 2,43 & 0,57 & 0 & 0,15 & 0,05 & 5,02 & 0 & 0 & 0,05 & 0 & 0,15 \\
\hline $\begin{array}{l}\text { MBZ } \\
26\end{array}$ & 7,8 & 15,2 & 12,47 & 9,86 & 11,51 & $\mathrm{X}$ & 0 & 0,13 & $\mathrm{x}$ & 3,97 & 0 & 0 & 0 & $\mathrm{X}$ & 2,6 \\
\hline $\begin{array}{l}\text { MBZ } \\
27\end{array}$ & 8,5 & 12,1 & 21,49 & 7,44 & 7,021 & 0 & 0 & 0 & $\mathrm{x}$ & 6,38 & 0 & 0 & 0,63 & 0 & 1,9 \\
\hline $\begin{array}{l}\text { MBZ } \\
28\end{array}$ & 6,9 & 14,8 & 15,25 & 2,48 & 5,67 & $\mathrm{X}$ & $\mathrm{X}$ & 0 & 0,35 & 17,38 & 1,41 & 0 & 0,17 & 0,17 & 0,35 \\
\hline $\begin{array}{l}\text { MBZ } \\
29\end{array}$ & 7,8 & 13,8 & 13,66 & 5,31 & 5,46 & $\mathrm{X}$ & $\mathrm{X}$ & 0 & 0,45 & 22,76 & 1,6 & 0 & 0,3 & 0,6 & 1,51 \\
\hline $\begin{array}{l}\text { MBZ } \\
30\end{array}$ & 0 & 0,6 & 0 & 14,84 & 13,55 & $\mathrm{X}$ & 2,58 & 6,45 & 0,96 & 0 & 0 & 0 & 1,9 & 0 & 3,2 \\
\hline
\end{tabular}

\section{Results}

\subsection{Lithostratigraphy}

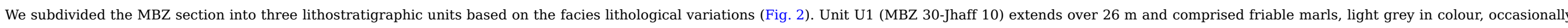

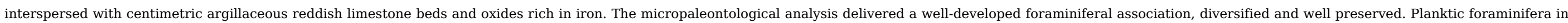




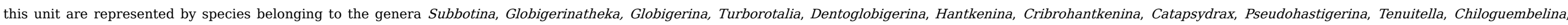

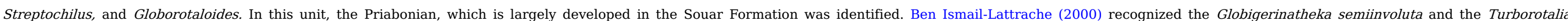
cerroazulensis Biozones and considered them as belonging to the Priabonian stage (see Fig. 3 ).

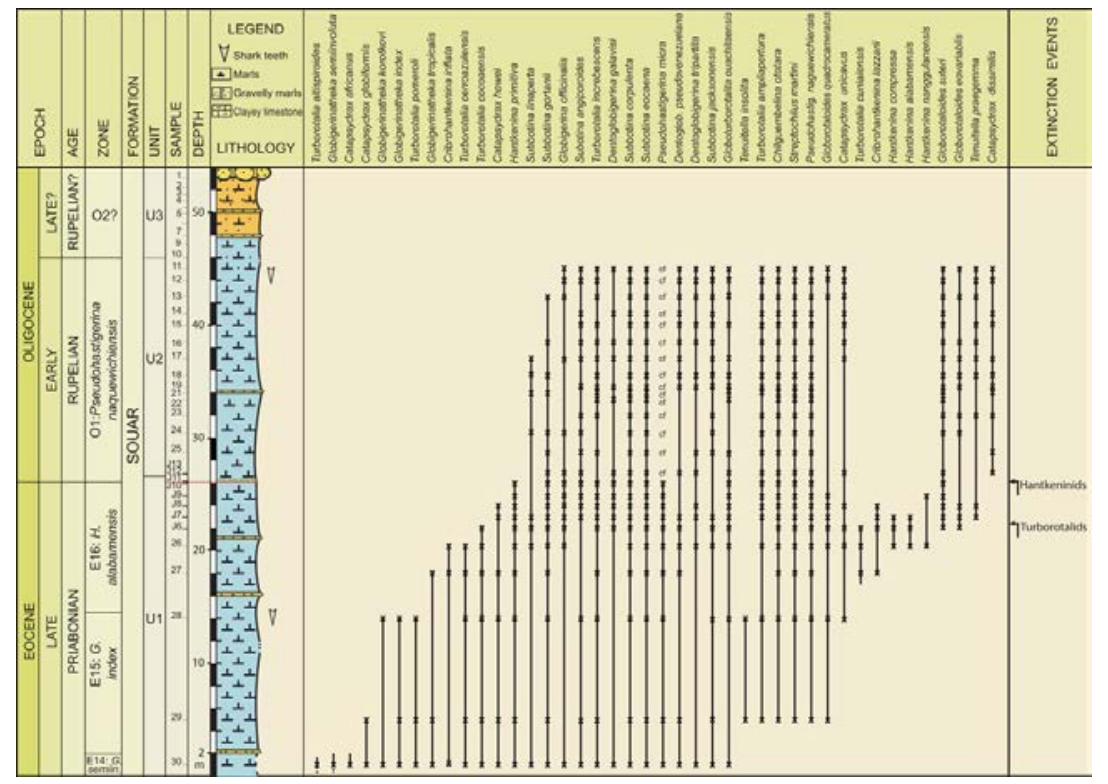

Fig. 2 Composite stratigraphical distribution of planktic foraminiferal species. alt-text: Fig. 2

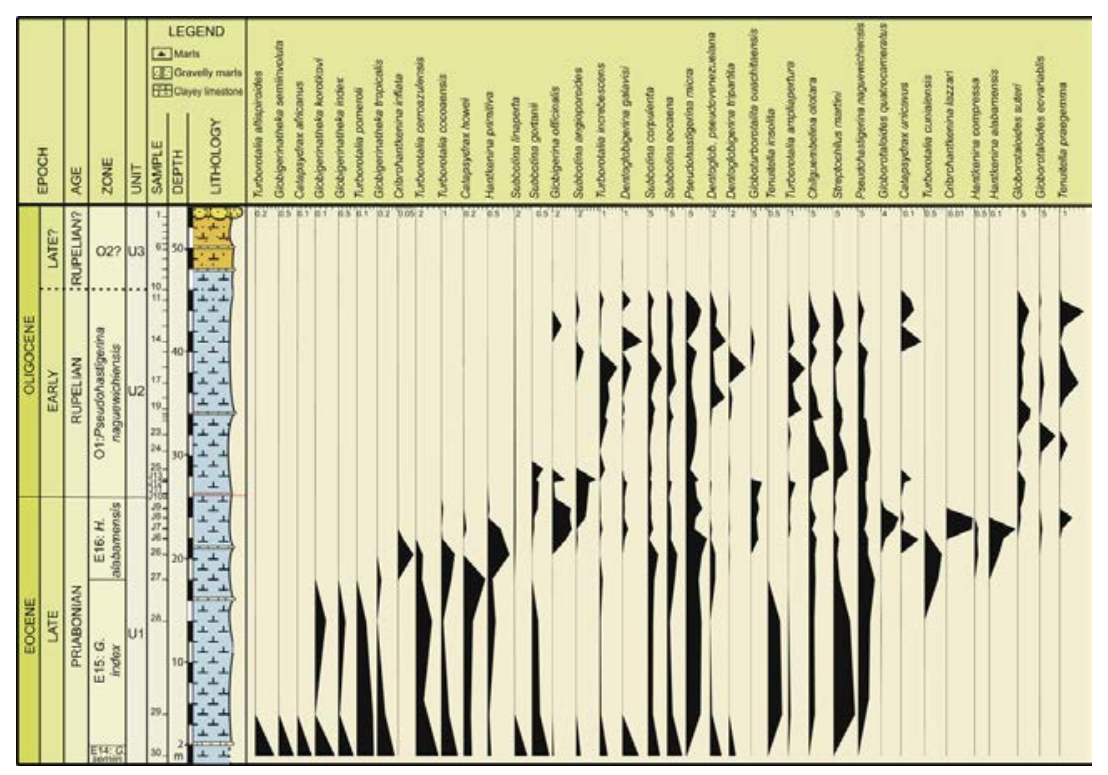

Fig. 3 Composite quantivative distribution of planktic foraminiferal species. 


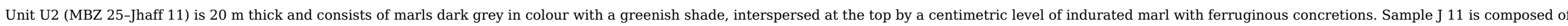

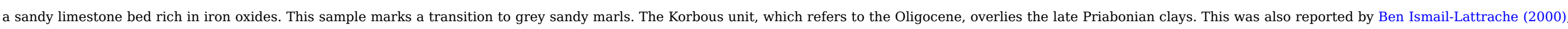

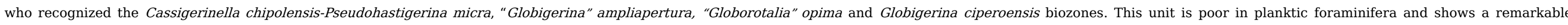

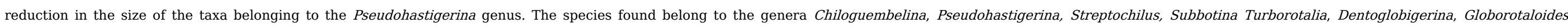

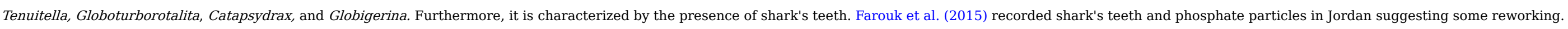

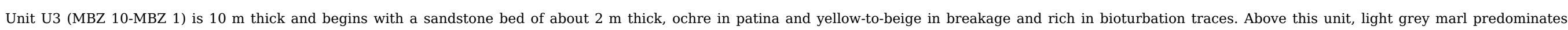

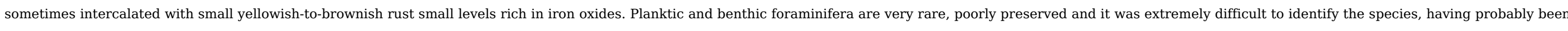

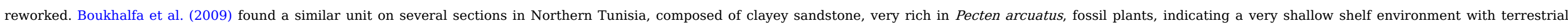
influence. Consequently, its age is very uncertain and is likely to be upper Rupelian or Chattian.

\subsection{Biostratigraphy}

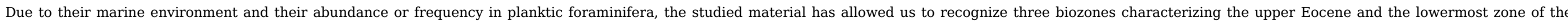
lower Oligocene (Fig. 2).

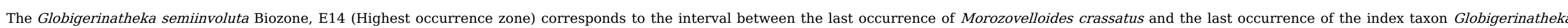

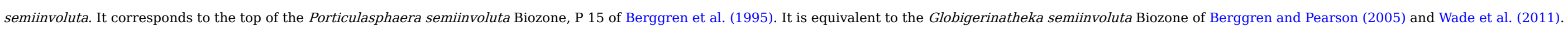

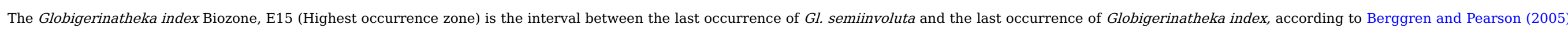

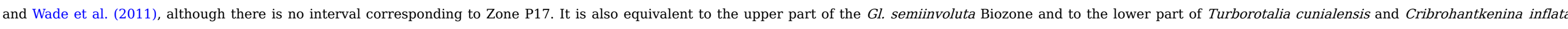
Biozone, P16 of Berggren et al. (1995).

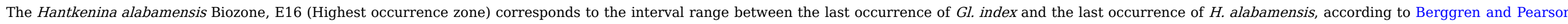

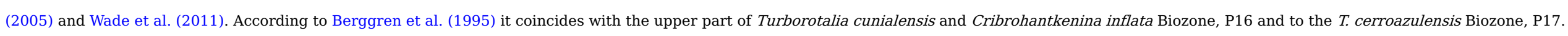

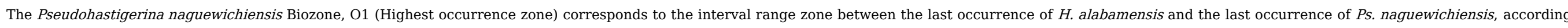
to Berggren and Pearson (2005) and Wade et al. (2011). It correlates to the Turborotalia cerroazulensis/Pseudohastigerina spp. Biozone, P18, according to Berggren et al. (1995).

\section{Discussion}

\subsection{Biostratigraphy}

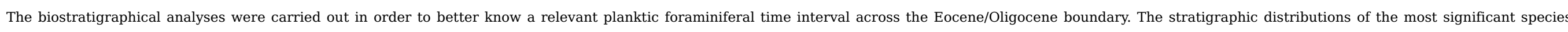
shown in Fig. 2 lead to the recognition of four biozones and their correlation to similar zones previously established by other authors.

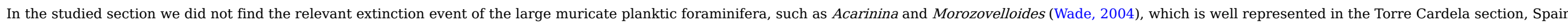

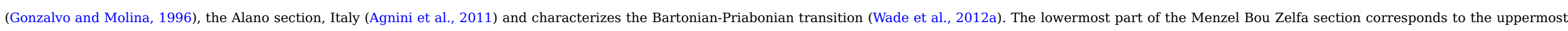
part of the E14 biozone (middle Priabonian).

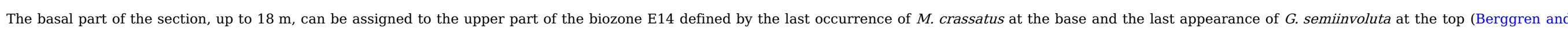

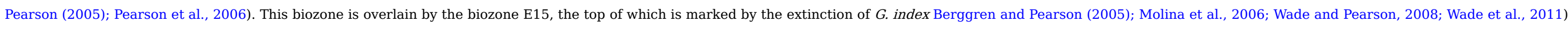

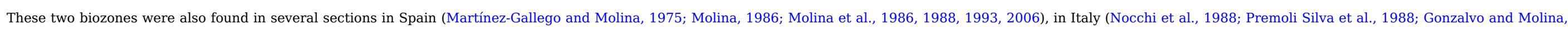
1992) and in Tanzania (Wade and Pearson, 2008; Pearson et al., 2008). 


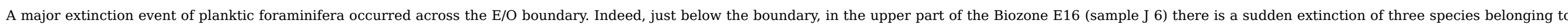

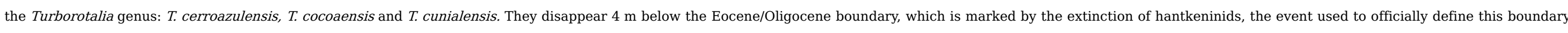

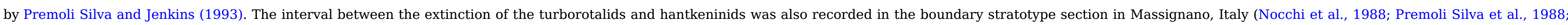
Gonzalvo and Molina, 1992) spanning only $0.4 \mathrm{~m}$. The extinction pattern across the E/O boundary can be considered a rapid mass extinction event (Molina, 2015).

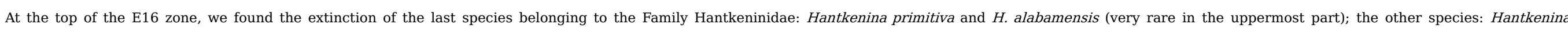

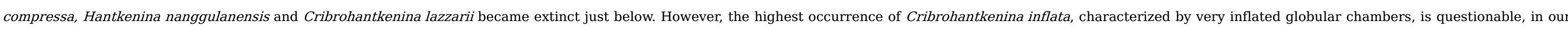

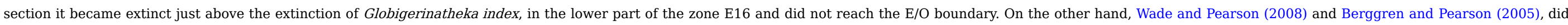
not recognize $C$. lazzarii, with the rugose square chambers; they considered it a junior synonym of $C$. inflata and indicated a simultaneous extinction of Hantkenina and Cribrohantkenina inflata.

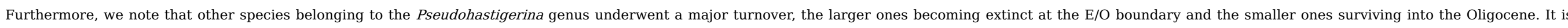

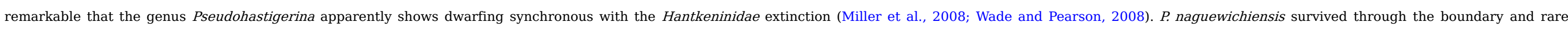

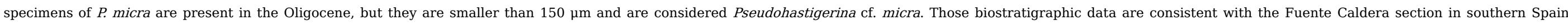
(Molina et al., 2006), the Tanzania section (Wade and Pearson, 2008; Pearson and Wade, 2015) and the Noroña section in Cuba (Molina et al., 2016).

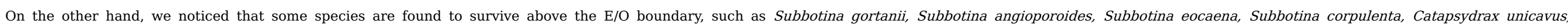

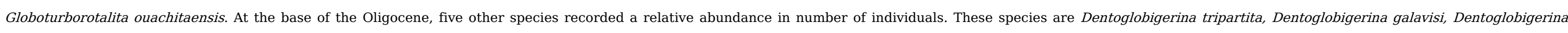
pseudovenezuelana, Turborotalia ampliapertura and Turborotalia increbescens (Table 1).

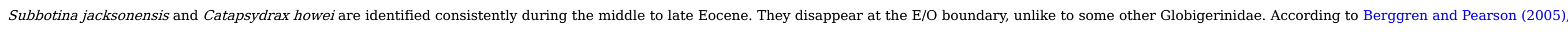

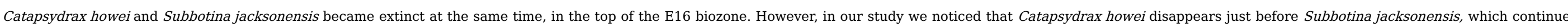
to the uppermost Eocene and became extinct at the same time as the Hantkeninidae.

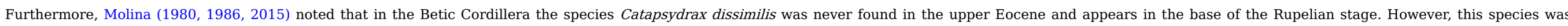

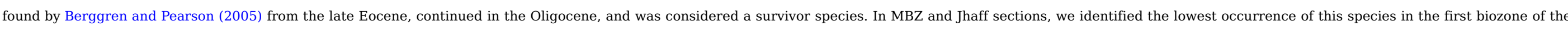
Oligocene, similar to what happened in the Betic Cordillera, Spain.

\subsection{Paleoecology}

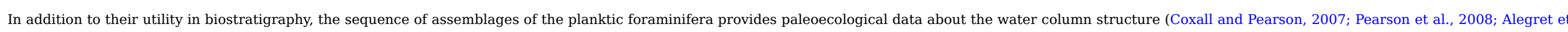

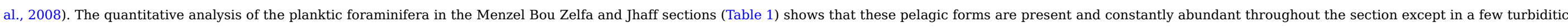

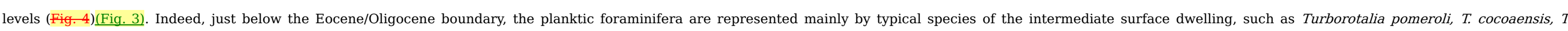

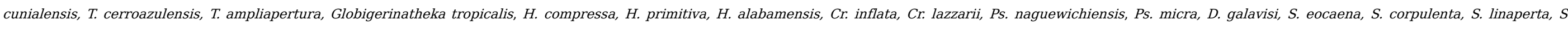

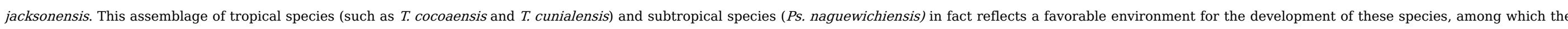
most abundant are S. corpulenta, S. eocaena, Ps. micra (about 10-15\%), which require intermediate dwelling (see Plate 1). 


\begin{tabular}{|c|c|c|c|c|c|c|c|c|}
\hline & TONE & $\begin{array}{l}\text { This study } \\
\text { Plankic foraminin }\end{array}$ & & ggren et al.(1995) & Berg & $\begin{array}{l}\text { ren \& Pearson } \\
(2005)\end{array}$ & & e et al. (2011) \\
\hline 产 & 01 & 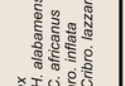 & $\mathbf{P 1}$ & T.ampliapertura IZ & 01 & $\begin{array}{l}\text { Hoz } \\
\text { Horchen }\end{array}$ & 01 & $\begin{array}{c}\text { guvewich } \\
\text { HOZ } \\
\text { s. }\end{array}$ \\
\hline & E16 & 然 & 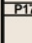 & Teent & E16 & $\begin{array}{l}\text { H.alabamensis } \\
\text { HOZ }\end{array}$ & E16 & $\begin{array}{l}\text { H.alabamensis } \\
\text { HOZ }\end{array}$ \\
\hline & E15 & & P1 & 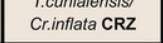 & $\begin{array}{l}\left.\right|_{E 15} \\
\mid\end{array}$ & G.index HOZ & |E15 & G.index $\mathrm{HOZ}$ \\
\hline & E14 & & $P 1$ & Po.seminvoluta IZ & E14 & $\begin{array}{c}\text { G.semminvoluta } \\
\text { HOZ }\end{array}$ & E14 & $\begin{array}{l}\text { G.seminivoluta } \\
\text { Hoz }\end{array}$ \\
\hline
\end{tabular}

Fig. 4 Biostratigraphic correlation across the Eocene/Oligocene boundary.

alt-text: Fig. 4

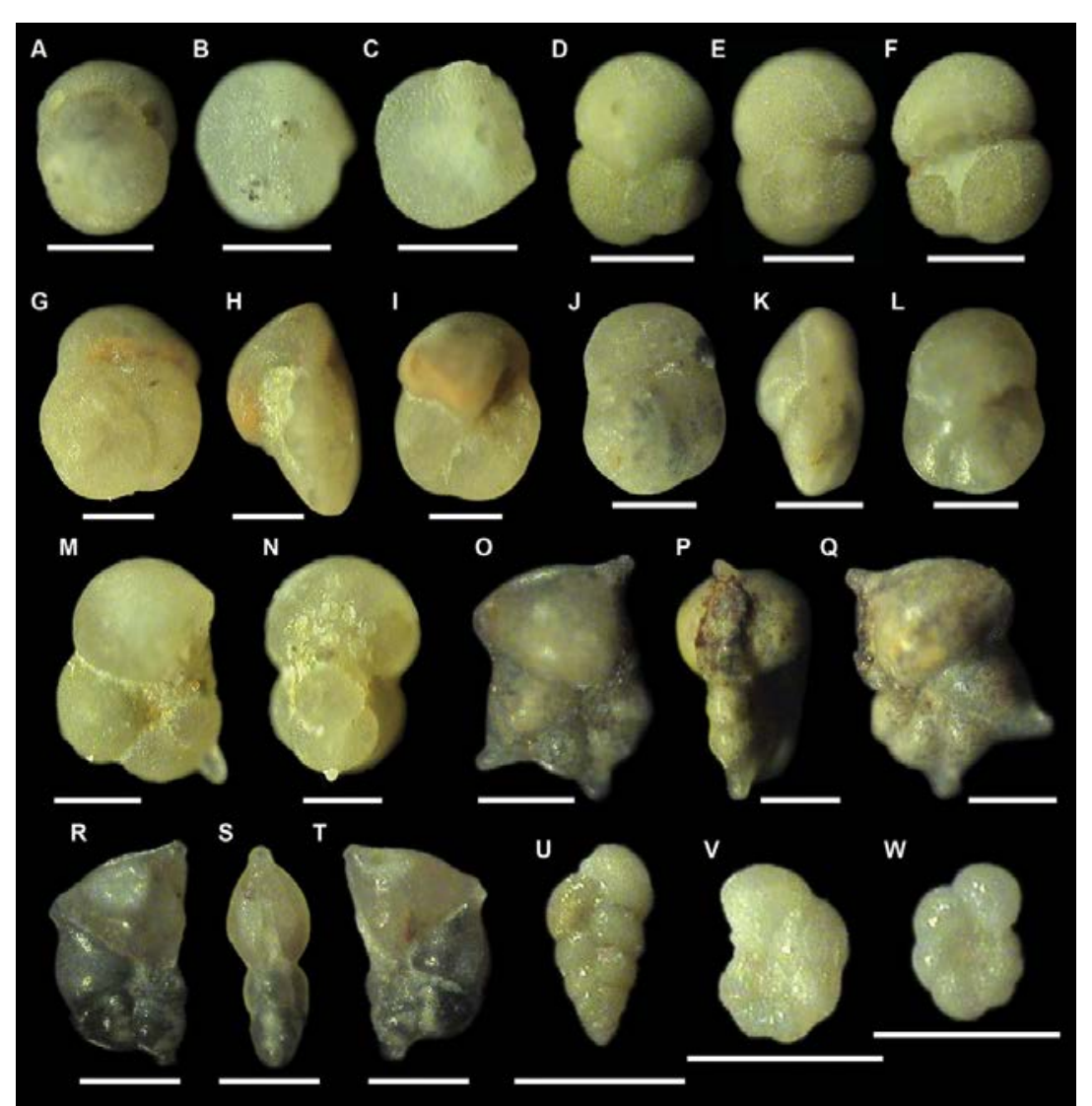

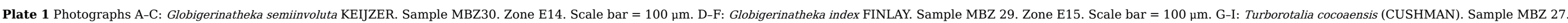

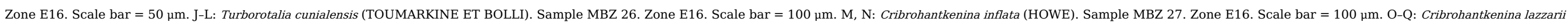

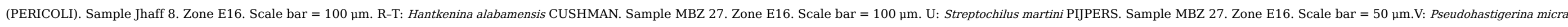
(COLE). Sample MBZ 12. Zone O1. Scale bar $=50 \mu \mathrm{m}$. W: Pseudohastigerina naguewichiensis MYATLIUK. Sample MBZ 12. Zone O1. Scale bar $=50 \mu \mathrm{m}$. 


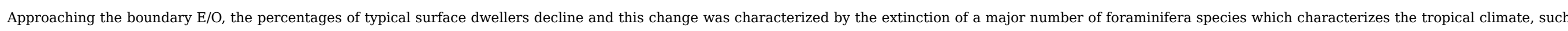

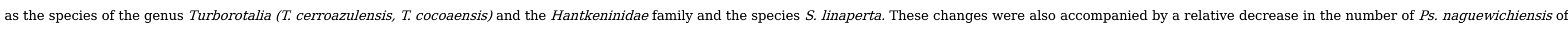

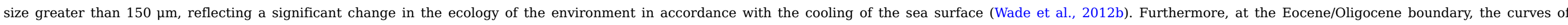
frequency show a slight decrease in the frequency of most forms except some species such as Ch. ototara and St. martini, which are considered indicators of stress (Gebhardt et al., 2013).

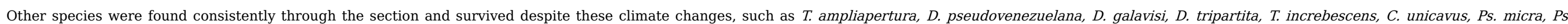

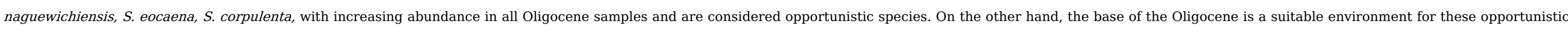

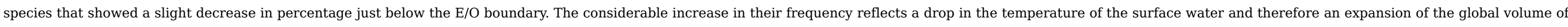

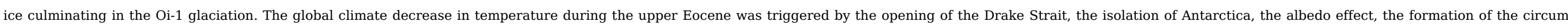
Antarctic current and the psychrosphere (Shackleton and Kennett, 1975; Kennett and Shackleton, 1976; Kennett, 1977; Barker and Thomas, 2004; Livermore et al., 2005).

\subsection{Chronostratigraphy}

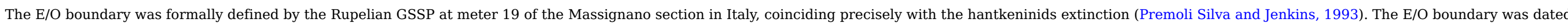

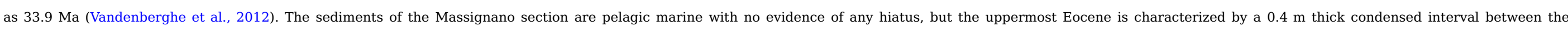

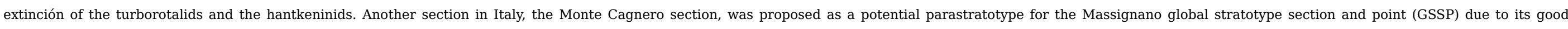
integrated stratigraphic and astrochronological calibration of the Eocene-Oligocene transition (Hyland et al., 2009), although the interval is similarly condensed.

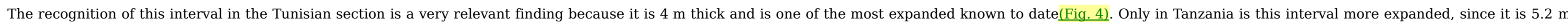

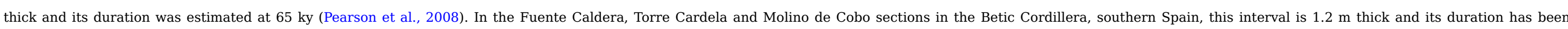
estimated at 40 ky (Molina, 2015). Nevertheless, in Jordan Farouk et al. $(2013,2015)$ found a hiatus missing this interval.

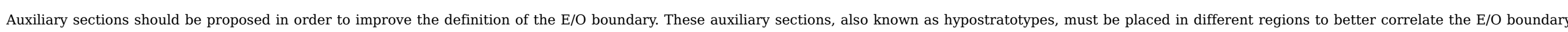

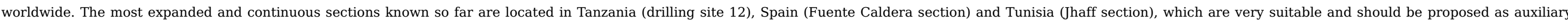
hypostratotypes.

\section{Conclusions}

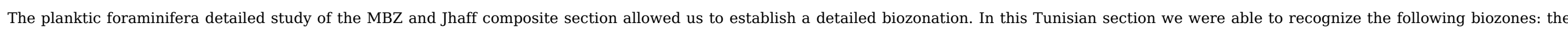

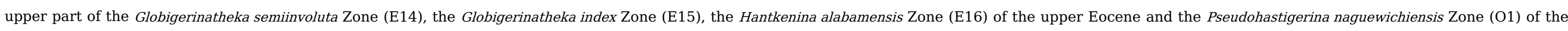
lower Oligocene.

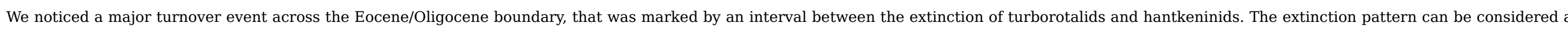

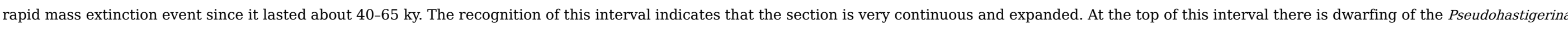
genus, with only the specimens no larger than $150 \mu \mathrm{m}$ surviving into the Oligocene.

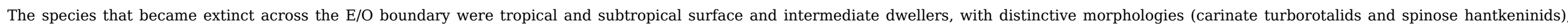

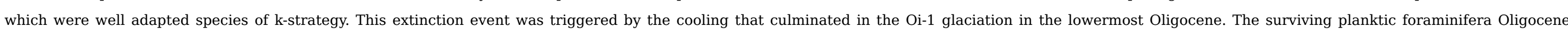
species were quite similar in morphology with globular chambers (globigerinids) and small planispiral pseudohastigerinids, which were mainly opportunistic species of r-strategy.

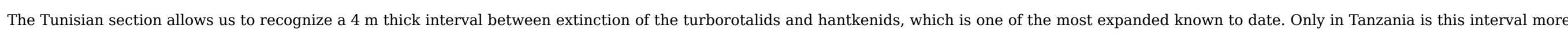

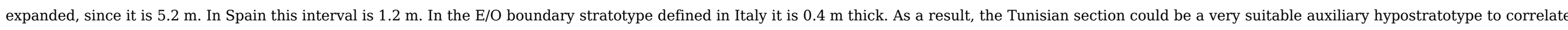
the E/O boundary worldwide. 


\section{Acknowledgements}

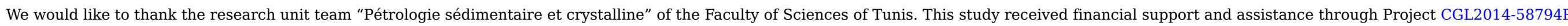
from the Spanish Ministry of Science and Technology (FEDER funds) and the Consolidated Group E05 from the Government of Aragón.

\section{References}

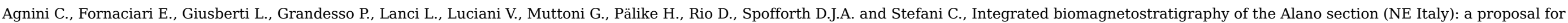
defining the middle late Eocene boundary, GSA Bull. 123 (5/6), 2011, 841-872.

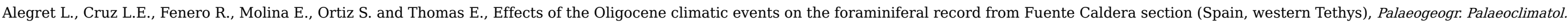
Palaeoecol. 269, 2008, 94-102.

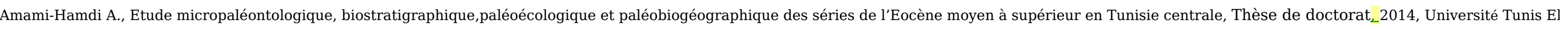
Manar, 230.

Barker P.F. and Thomas E., Origin, signature and paleoclimatic influence of the Antarctic circumpolar current, Earth-Science Rev. 66, 2004, 143-162.

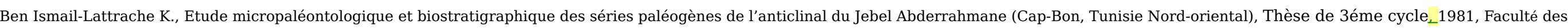
sciences de Tunis.

Ben Ismail-Lattrache K., Précision sur le passage Lutétien/Barthonien dans les dépôts éocènes moyens en Tunisie Centrale et Nord-orientale, Rev. Micropaléontologie 43, 2000, 3-16.

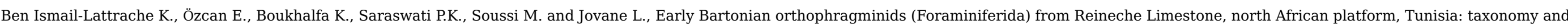
paleobiogeographic implications, Geodin. Acta 2014, http://dx.doi.org/10.1080/09853111.2013.858950

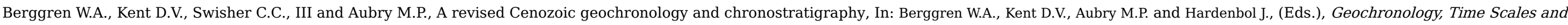
Global Stratigraphic Correlation, vol. 54, 1995, Society of Economic Paleontologists and Mineralogists (SEPM) (Society for Sedimentary Geology) SEPM, 129-212, Special Publication.

Berggren W.A. and Pearson P.N., A revised tropical and subtropical Paleogene planktonic foraminiferal zonation, J. Foraminifer. Res. 35, 2005, 279-298.

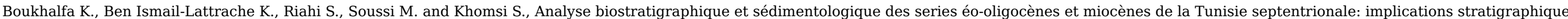
et géodynamiques, Comptes Redus Geosci. 341, 2009, 49-62.

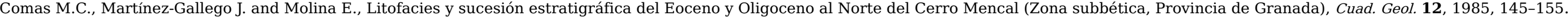

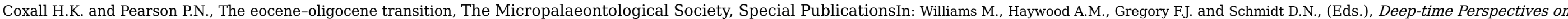
Climate Change: Marrying the Signal from Computer Models and Biological Proxies, 2007, The Geological Society; London, 351-387.

Farouk S., Ahmad F. and Smadi A.A., Stratigraphy of the middle eocene-lower Oligocene successions in northwestern and eastern Jordan, J. Asian Earth Sci. 73, 2013 , 396-408.

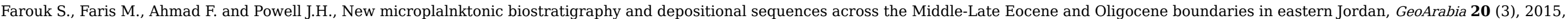
$145-172$.

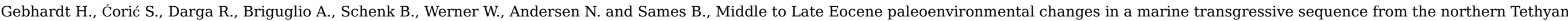
margin (Adelholzen, Germany), Austrian J. Earth Sci. 106 (2), 2013, 45-72.

Gonzalvo C. and Molina E., Bioestratigrafía y cronoestratigrafía del tránsito Eoceno-Oligoceno en Torre Cardela y Massignano (Italia), Rev. Española Paleontol. 7, 1992, 109-126.

Gonzalvo C. and Molina E., Bioestratigrafía y Cronoestratigrafía del tránsito Eoceno Medio-Eoceno Superior en la Cordillera Bética, Rev. Española Micropaleontol. 28 (2), 1996, 25-44. 
Hartenberger J.L., An Asian grande coupure, Nature 394, 1998, 321.

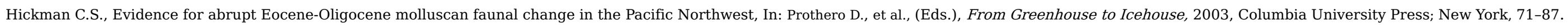

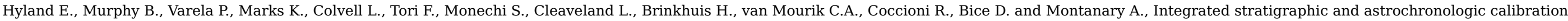

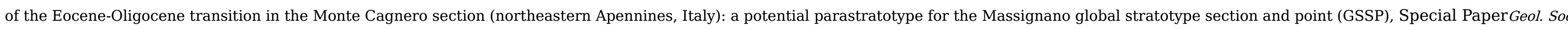
Am. 452, 2009, 303-322.

Kennett J.P., Cenozoic evolution of Antarctic glaciations, the circum-Antarctic ocean and their impact on global paleoceanography, J. Geophys. Res. 82, 1977, 3843-3860.

Kennett J.P. and Schackleton N.J., Oxygen isotopic evidence for the development of the psychrosphere 38 Myr ago, Nature 260, 1976, 513-515.

Livermore R., Nankivell A., Eagles G. and Morris P., Paleogene opening of Drake passage, Earth Planet. Sci. Lett. 236, 2005, 459-470.

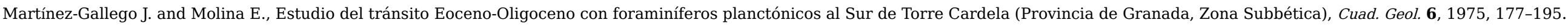

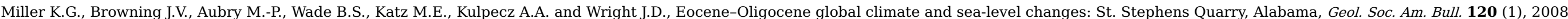
34-53.

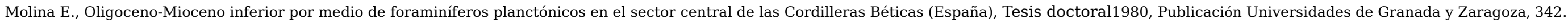

Molina E., Description and biostratigraphy of the main reference section of the Eocene/Oligocene boundary in Spain: Fuente Caldera section, Dev. Paleontology Stratigr. 9, 1986, 53-63.

Molina E., Evidence and causes of the main extinction events in the Paleogene based on extinction and survival patterns of foraminifera, Earth-Science Rev. 140, 2015, 166-181.

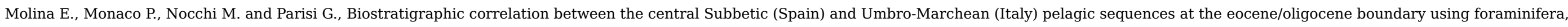
Dev. Paleontology Stratigr. 9, 1986, 75-85.

Molina E., Keller G. and Madile M., Late Eocene to Oligocene events: Molino de Cobo, Betic Cordillera, Spain, Rev. Española Micropaleontol. 20, 1988, $491-514$.

Molina E., Gonzalvo C. and Keller G., The Eocene-Oligocene planktic foraminiferal transition: extinctions, impacts and hiatuses, Geol. Mag. 130 (4), 1993, 483-499.

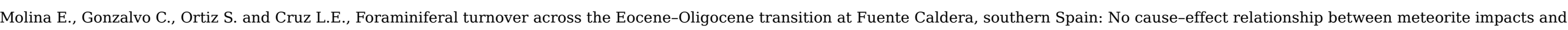
extinctions, Mar. Micropaleontol. 58, 2006, 270-286.

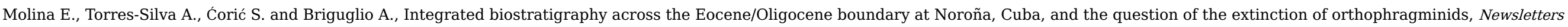
Stratigr. 49 (1), 2016, 27-40.

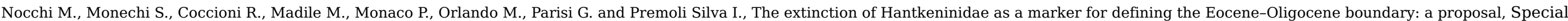

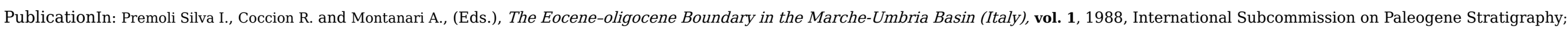
Ancona, 249-252.

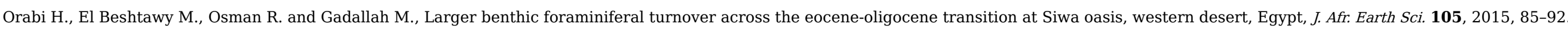

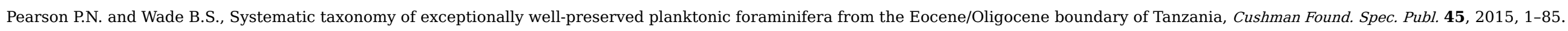

Pearson P.N., Olsson R.K., Huber B.T., Hemleben C. and Berggren W.A., (Eds.), Atlas of Eocene Planktonic Foraminifera, 41, 2006, Cushman Foundation Special Publication, 514.

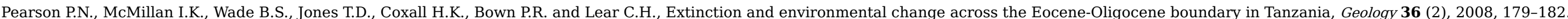
Premoli Silva I. and Jenkins G., Decision on the Eocene Oligocene boundary stratotype, Episodes 16 (3), 1993, 379-382.

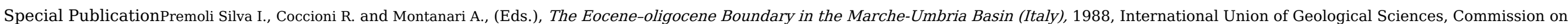


Stratigraphy; Ancona, 268.

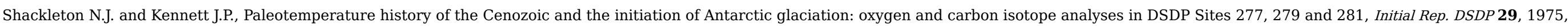
743-755.

Vandenberghe N., Hilgen F.J. and Speijer R.P., The Paleogene period, In: Gradstein F.M., et al., (Eds.), The Geologic Time Scale 2012, 2012 , Elsevier, 855-921.

Wade B.S., Planktonic foraminiferal biostratigraphy and mechanisms in the extinction of Morozovella in the late middle Eocene, Mar. Micropaleontol. 51, $2004,23-38$.

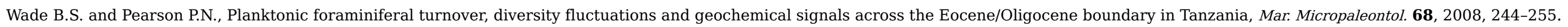

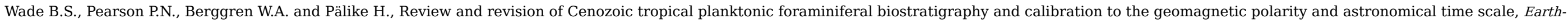
Science Rev. 104, 2011, 111-142.

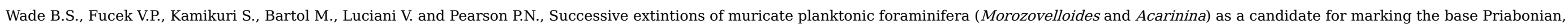
Newsletters Stratigr. 45 (3), 2012a, 245-262.

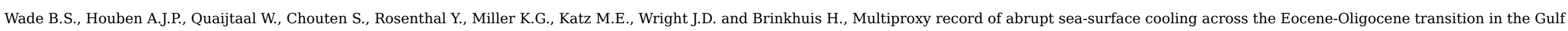
of Mexico, Geology 40 (2), 2012b, 159-162.

\section{Highlights}

- Planktic foraminifera E14, E15, E16, O1 Zones are identified across the E/O boundary.

- The section is correlated with others in Tanzania, Spain and the E/O GSSP in Italy.

- Turborotalids and hantkeninids underwent a rapid mass extinction across the E/O.

- The extinction event at the E-O transition is recorded in a very expanded interval.

- The Tunisian section is a very suitable auxiliary section for the E/O boundary.

\section{Queries and Answers}

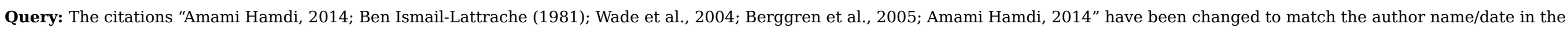
reference list. Please check.

Answer: Ok

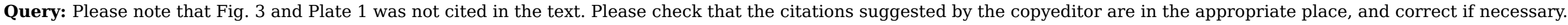
Answer: I changed the place of Fig. 3 and Fig. 4

Query: Please provide the grant number for ' Government of Aragón' if any.

Answer: The number is E05

Query: Please confirm that given names and surnames have been identified correctly.

Answer: Yes 
